\title{
Percepção dos utilizadores sobre o software de snálise qualitativa webQDA
}

\author{
User's perception about the qualitative analyses software webQDA
}

\section{Percepción de usuarios acerca de software de análisis cualitativo webQDA}

\author{
Dayse Neri de Souzal \\ Francislê Neri de Souza ${ }^{2}$ \\ António Pedro Costa ${ }^{3}$ \\ http://dx.doi.org/10.5216/33270
}

\section{Resumo}

A investigação de natureza quantitativa revela-se limitadapara responder algumas das questões das ciências humanas e sociais.Por outro lado, os investigadores reconhecem cada vez mais que a investigação qualitativa tem uma importância crucial para muitas das questões de investigação que gostaríamos aprofundar. A qualidade e o número de trabalhos nesta área são consideráveis, mas são muitos os desafios a enfrentar. Um dos desafios que discutiremos neste artigo é ao nível da formação de novos investigadores na conjugação de técnicas de análise e um software disponível para potencializar esta mesma análise. Assim, neste trabalho, apresentamos as percepções de investigadores sobre a utilização do software webQDA no contexto de uma formação avançada. Para além do perfil dos participantes, apresentamos também uma avaliação sobre o programa de formação em si. O Web Qualitative Data Analysis (webQDA) é um software de apoio à análise qualitativa que pode ser acedido através da internet, proporcionando um ambiente seguro de trabalho colaborativo (www.webqda.com). Recorremos a uma análise qualitativa de questões abertas com a utilização do próprio webQDA, que foram recolhidas através de um inquérito por questionário. Os resultados apontam para uma percepção positiva dos formandos acerca da utilização do webQDA e da própria formação. No entanto, apontam limitações técnicas que na versão inicial do sistema ainda não se encontravam disponíveis, tais como: exportar tabelas para Excel, imprimir e exportar dados textuais e trabalhar offline. Quanto à formação, sugerem que é necessário mais tempo para a dimensão do questionamento e cruzamentos dos dados com apoio do webQDA. Foi possível perceber também que a utilização do software é um elemento dinamizador de novas compreensões e aprofundamentos sobre o processo de análise qualitativa de dados.

Palavras-chave: webQDA. Análise de dados. Avaliação de Software. Formação.

\begin{abstract}
The nature of quantitative research reveals is limited to answering some of the questions the human and social sciences. On the other hand, researchers increasingly recognize that qualitative research is of crucial importance to answer research questions that we would like to deepen. The quality and number of works in this area are considerable, but there are many challenges to face. One of the

\footnotetext{
${ }^{1}$ Doutora em Ciências da Educação, licenciada em Ciências Sociais. Universidade de Aveiro. Departamento de Educação e CIDTFF, Centro de Investigação Didáctica e Tecnologia na Formação de Formadores. Portugual, Aveiro. E-mail: dayneri@ua.pt

2 Doutor em educação em Ciência. Licenciado em Química. Universidade de Aveiro. Departamento de Educação do Centro de Investigação Didáctica e Tecnologia na Formação de Formadores (CIDTFF). Portugual, Aveiro. E-mail: fns@ua.pt

${ }^{3}$ Doutor em multimédia em Educação. Universidade de Aveiro. Departamento de Educação do Centro de Investigação Didáctica e Tecnologia na Formação de Formadores (CIDTFF). Portugual, Aveiro. E-mail: pcosta@ludomedia.pt
}

Comun. \& Inf., Goiânia, GO, v. 17, n. 2, p. 104-118, jul./dez. 2014. 
challenges that we discuss in this paper is the level of training of new researchers toward combination of analysis techniques and software available to enhance this same analysis. In this work, we present the perceptions of researchers on the use of webQDA software in the context of advanced training. Beyond the participants' profile, we also present an evaluation of the training program itself. The Web Qualitative Data Analysis (webQDA) is software to support qualitative analysis that can be accessed through the internet, providing a secure collaborative work environment (www.webqda.com). We use a qualitative analysis of open-end questions using the own webQDA software, which were collected through a questionnaire. The results point to a positive perception of the trainees on the use of webQDA and training itself. However, technical limitations are indicated that in the initial version of the system were not yet available, such as: export tables to Excel, print and export text data and work offline. As for training, suggest that is necessary more time to the Questioning dimension and triangulation of data with support of webQDA. Was possible seen also that the use of software is a driving force for new understandings and insights about the process of qualitative analysis of data.

Keywords: Component. Formatting. Style. Styling. Headings.

\section{Resumen}

La investigación de carácter cuantitativo parece limitada para responder a algunas cuestiones de 1 ciencias humanas y sociales. Los investigadores reconocen que la investigación cualitativa tiene una importancia crucial para muchos de los temas de investigación que se pretende impulsar y desarrollar. La calidad y el número de trabajos en este ámbito son importantes, pero hay muchos retos que afrontar. Uno de los retos, en este artículo, es el nivel de formación de nuevos investigadores en las técnicas de análisis y un software disponible para potenciar el análisis. Por este motivo, presentamos las percepciones de los investigadores en el uso del software webQDA en formación avanzada. Además del perfil de los participantes, se presenta una evaluación del programa de formación. La Web Análisis de Datos Cualitativos (webQDA) es un software para apoyar el análisis cualitativo que se tiene acceso a través de internet, proporcionando un entorno seguro de trabajo colaborativo (www.webqda.com). Utilizamos un análisis cualitativo de preguntas abiertas y cerradas con el uso de webQDA, a través de una encuesta mediante cuestionario. Los resultados apuntan a una percepción positiva de los alumnos sobre el uso de webQDA y de su propia formación, más limitaciones técnicas que en la primera versión del sistema todavía no estaban disponibles, tales como: exportar tablas para Excel, imprimir y exportar datos de texto y trabajar sin conexión. Sugieren que se necesita de más tiempo para el tamaño de cuestionamiento y relación de datos con el apoyo de webQDA. Es posible darse cuenta de que el uso del software es una de las fuerzas que impulsan los nuevos entendimientos y reflexiones sobre el proceso de análisis cualitativo de los datos.

Palabras-clave: WebQDA. Análisis de los datos. Software de evaluación. Formación.

\section{INTRODUÇÃO}

investigação qualitativa tem alcançado relevância em diversas áreas de
investigação. Mesmo em áreas como a psicologia, onde a investigação
quantitativa predomina, alguns investigadores tem apontado que: "The data that

we have obtained seem to indicate that qualitative research publications will continue increasing in the coming years". (CARRERA-FERNÁNDEZ; GUÀRDIA-OLMOS; PERÓCEBOLLERO, 2014). Este é um indicador importante que aponta para uma maior massa crítica de investigadores a se debruçar nesta área de investigação.

Comun. \& Inf., Goiânia, GO, v. 17, n. 2, p. 104-118, jul./dez. 2014. 
No entanto, existe ainda muitos desafios atuais para a investigação qualitativa, dos quais destacamos aqui dois deles: i) desafios ao nível das terminologias, técnicas e instrumentos de análise, e ii) desafios ao nível da formação de novos investigadores na conjugação destas técnicas e os pacotes de software que existem disponíveis para potencializar esta mesma análise.

No âmbito destes desafios, há na própria literatura da área transformações ao nível do jargão específico, seu significado e funções. Também por isso, não é incomum a utilização mal aplicada de alguma terminologia da investigação qualitativa. Assim, perante esta realidade, os investigadores indicam que: "The results showed that "textual analysis" cannot be considered a specific methodology and so the use of this term as a particular technique could be misleading. This highlights the need to create a common vocabulary to facilitate the systematization and dissemination of work done with qualitative methods" (CARRERAFERNÁNDEZ; GUÀRDIA-OLMOS; PERÓ-CEBOLLERO, 2013). Porém os desafios não se restringem à terminologia da investigação qualitativa, apenas pode indicar a falta de domínio e integração de técnicas, tecnologias e níveis de reflexões mais atualizadas e aprofundada.

Quando se procura integrar as tecnologias, especificamente software para análise de dados na investigação qualitativa ( $Q D A S$ - Qualitative Data Analysis Software), o segundo desafio mencionado é a formação de investigadores. Geralmente os investigadores iniciantes perguntam: Qual QDAS devo usar? E o que um QDAS poderá contribuir na minha análise? Quando antes deveriam perguntar: Quais são as tarefas analíticas que necessito desempenhar? Como poderei usar o QDAS para apoiar meu estudo nos diferentes caminhos metodológicos que escolhi? Todos os pacotes de software que estão disponíveis hoje, incluído o webQDA, são ferramentas que auxiliam a análise, não "faz" a análise no lugar do investigador. O poder analítico e crítico continua centrado no investigador e na sua rede análise.

Neste sentido, as recentes investigações sobre análise qualitativa de dados com software indicam que é mais crítico as competências dos investigadores e suas escolhas metodológicas do que a seleção de um software QDA específico para determinar a qualidade da análise (LINDA S.; KRISTI; GREGORIO, 2014). No entanto, todos reconhecem novas oportunidades e o desenvolvimento de novas competências de análise que as ferramentas QDA proporcionam. Portanto, é importante dedicar tempo para analisar as vantagens e desafios analíticos que o webQDA pode oferecer.

Neste artigo tencionamos portanto analisar a opinião de 59 investigadores na sua maioria frequentando mestrados e doutoramentos e que participaram da formação sobre 
análise qualitativa com apoio do webQDA. Iremos aprofundar uma análise quantitativa já realizada (NERI DE SOUZA; COSTA; NERI DE SOUZA, 2012a), mas agora com dados não-numéricos e não-estruturados destes formandos numa análise qualitativa realizada com o próprio webQDA. Nesta análise, além de cruzar com os resultados da análise quantitativa anterior procuraremos compreender a percepção destes formandos em duas perspectivas: i) o software webQDA em si; ii) a formação sobre o webQDA.

\section{A IMPORTÂNCIA dA AVALIAÇÃo PARA A MELHORIA dOS PRODUTOS E DOS PROCESSOS: AVALIAÇÃO DE SOFTWARE}

A avaliação de software centrada no utilizador serve a três objetivos: i) suportar à tomada de decisões, ii) detetar problemas e iii) verificar a qualidade do software ("Readerfocused text evaluation. An overview of goals and methods," 1997). Estes objetivos fazem da avaliação uma valiosa ferramenta para as equipas multidisciplinares, uma vez que justifica os seus esforços, melhorando o software e apoiando a equipa de desenvolvimento nas tomadas de decisão relativamente à versão do software a implementar (COSTA, REIS; LOUREIRO, 2014).

Quando mencionamos os termos "aferição da qualidade de uso de um software" e ambicionámos alcançá-la, a usabilidade é um dos critérios mais referidos na literatura. A usabilidade diz respeito a um conjunto de atributos que determinado software deverá conter de forma a que os utilizadores consigam atingir os seus objetivos com eficiência, eficácia e satisfação de uso em determinado contexto de utilização(ISO/IEC9126, 1999). As métricas para avaliar a Usabilidade são:

- Eficácia: precisão e perfeição com que os utilizadores atingem os objetivos;

- Eficiência: recursos despendidos relativamente à precisão e perfeição com que os utilizadores atingem os objetivos;

- Satisfação de uso: conforto e atitudes positivas relativas ao uso do software;

- Contexto de utilização: que compreende os utilizadores, as tarefas, os equipamentos (hardware, software e recursos), o ambiente físico e social em que o software é utilizado.

Na seção seguinte apresentamos a estrutura do inquérito por questionário e os objetivos do curso de formação que serviu de suporte para a recolha de dados. 


\section{METODOLOGIA}

Esta investigação foi realizada no âmbito de formações sobre "Análise Qualitativa com o Apoio do Software webQDA" a formandos de diversas áreas científicas. Intentamos aferir a opinião destes participantes acerca das potencialidades do uso desta ferramenta, e da própria formação em si. Para isso, recorremos a uma metodologia qualitativa exploratória com base num questionário com questões fechadas e abertas.

Neste artigo analisamos as respostas abertas do questionário que foi dividido em três partes, a saber: a) perceção sobre a formação, b) a utilização do software webQDA e c) o perfil dos formandos. Para responder ao questionário, foi enviado o link de acesso online a todos os formandos que participaram nas diferentes edições do curso de Formação. Cruzamos os resultados da análise das questões abertas com o que estes participantes assinalaram nas questões fechadas. Para a análise das questões seguimos os passos da análise de conteúdo (NERI DE SOUZA, COSTA \& NERI DE SOUZA, 2012; NERI DE SOUZA, COSTA, \& MOREIRA, 2011; LINHARES \& NERI DE SOUZA, 2014). Foi utilizado o próprio software webQDA (última versão disponível online) como suporte informático para a análise.

Acerca do perfil dos formandos, os dados indicam que tinham entre os 23 e 62 anos de idade, $83,1 \%(n=49)$ são do sexo feminino e $17 \%(n=10)$ do sexo masculino. Dos 59 respondentes, $41 \%(n=26)$ estavam a realizar o mestrado e $46 \%(n=27)$ doutoramentos. Relativamente às áreas científicas dos formandos, os dados revelam que 56\% (n=33) são da área de educação (ensino e didática). Percebemos também que 34\% ( $\mathrm{n}=20)$ desenvolvem estudos na área da investigação qualitativa entre 1 a 2 anos, 71,2\% (n=42) dedicam tempo parcial ao trabalho de investigação e $90 \%(n=53)$ dos formandos nunca utilizaram outro software de suporte à investigação qualitativa ou à técnica de análise de conteúdo.

Acerca das edições dos cursos, todas têm a duração de 8 horas e realiza-se normalmente num único dia. O curso de formação é suportado sob os seguintes objetivos:

- aprofundar conhecimentos no sentido de auxiliar os investigadores a lidar com dados não numéricos e não estruturados em análise qualitativa;

- capacitar para a utilização de software de análise qualitativa webQDA;

- discutir como esta ferramenta (webQDA) pode ser usada em diversas metodologias de investigação e conjuntamente com outras ferramentas;

- desenvolver um projeto que sirva de modelo e de base de aprendizagem das funções disponíveis no webQDA. 
Para tornar possível o atingir destes objetivos, a formação é estruturada da seguinte forma:

- Razões para a utilização de um software de análise qualitativa; expetativas não confirmáveis para o uso do webQDA; organização e potencialidades do webQDA; introdução das ações e comandos do webQDA; Sistema de Dados (Fontes); casos e atributos.

- Procedimentos de codificação; Sistema de Categorias (Nós); como relacionar dados e Nós para construir modelos.

- A importância de questionar para extrair do webQDA as informações de que se necessita (Sistema de Questionamento). Qual é o significado das análises efetuadas e dos resultados obtidos? Qual a consistência da codificação ao longo da análise dos dados?

- Exploração de padrões através da geração de matrizes; elementos obtidos do webQDA que apoiam a escrita dos resultados da análise; discussão dos projetos individuais e/ou de grupo.

Naturalmente, compreendemos que muitos destes objetivos de aprendizagem e desenvolvimento de competências serão iniciados na formação, que necessitará de muito esforço e experiência dos formandos para se aprofundarem de forma completa e avançada na análise qualitativa.

\section{FUNDAMENTO ESTRUTURAL E PROCESSUAL DO WEBQDA}

Antes de iniciarmos propriamente a apresentação dos resultados consideramos que é importante para o leitor que ainda não conhece o software online webQDA ${ }^{\circledR}$ (www.webqda.com), ter uma compreensão geral sobre as bases estruturais e processuais que fundamentam a sua utilização. Neri de Souza, Neri de Souza, Costa, \& Moreira (2013) resumem a estrutura do webQDA tem três componentes básicas: i) Fontes, ii) Codificação e iii) Questionamento (Figura 1). Cada uma destas partes tem um conjunto de ferramentas e funções que se articulam para apoiar o investigador na sua análise. 
Figura 1 - Fundamento Estrutural do webQDA

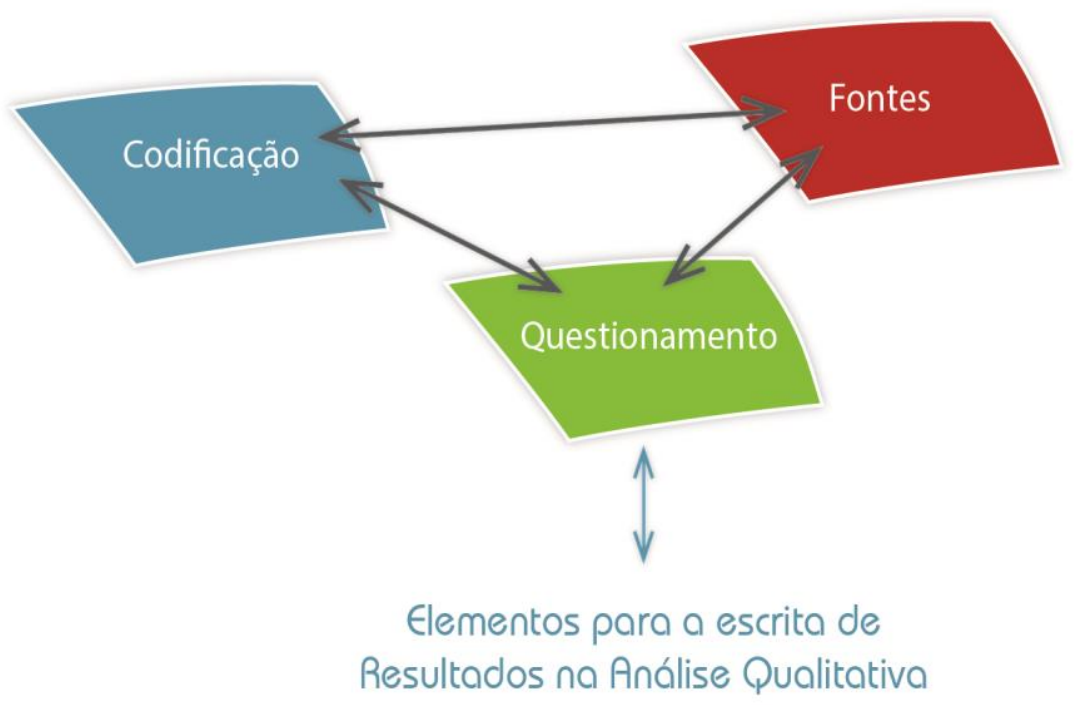

Nas "Fontes" o utilizador coloca todos os seus dados não-numéricos e nãoestruturados (textos, áudio, vídeo e imagens), provenientes das diversas fontes de dados recolhidas durante todo o processo. Isso quer dizer que o sistema pode ser usado desde o primeiro momento do processo de investigação e ser alimentado em estágios cíclicos ou ser alimentado por completo na fase final da investigação. $\mathrm{Na}$ parte das "Codificações" é disponibilizado para o investigador as ferramentas para que ele mesmo faça a codificação dos dados. Nem o webQDA, nem nenhum outro sistema de codificação de dados atualmente disponível tem mecanismo de inteligência artificial para codificar os dados. Assim, é sempre o investigador com o quadro teórico, contextual e relacional que tem condição para codificar plenamente os seus dados.

$\mathrm{Na}$ parte do "Questionamento" o webQDA disponibiliza um conjunto de ferramentas para fazer busca, cruzar e sintetizar na procura de padrões dos dados codificados e/ou ainda codificar em função de perguntas que o investigador possa fazer ao seu sistema codificado.

Estas três partes são dinâmicas e flexíveis para se adaptar aos diversos modelos e processos de análise qualitativa de dados. É da interação destas três partes que o webQDA fornece elementos que irá apoiar o investigador na escrita de resultados, no processo de validação e de articulação com outros investigadores (ver Figura 1).

Outra importante visão sobre o webQDA, que também está relacionada com os objetivos da formação e da análise que fazemos neste artigo, está ancorada na parte 
processual da utilização do sistema. O processo de utilização do webQDA coincide com o próprio processo de análise de dados numa investigação qualitativa. Por isso, tem todas as dificuldades inerentes a este processo do qual o sistema informático se propõe a otimizar no sentido de um tornar mais eficiente, eficaz e transparente. Na Figura 2, Neri de Souza, Costa e Moreira (F. Neri de Souza et al., 2013) apresentam um resumo esquemático do processo de utilização do webQDA em duas fases: i) configuração do projeto e ii) abstração do projeto.

Figura 2 - Fundamento Processual do webQDA

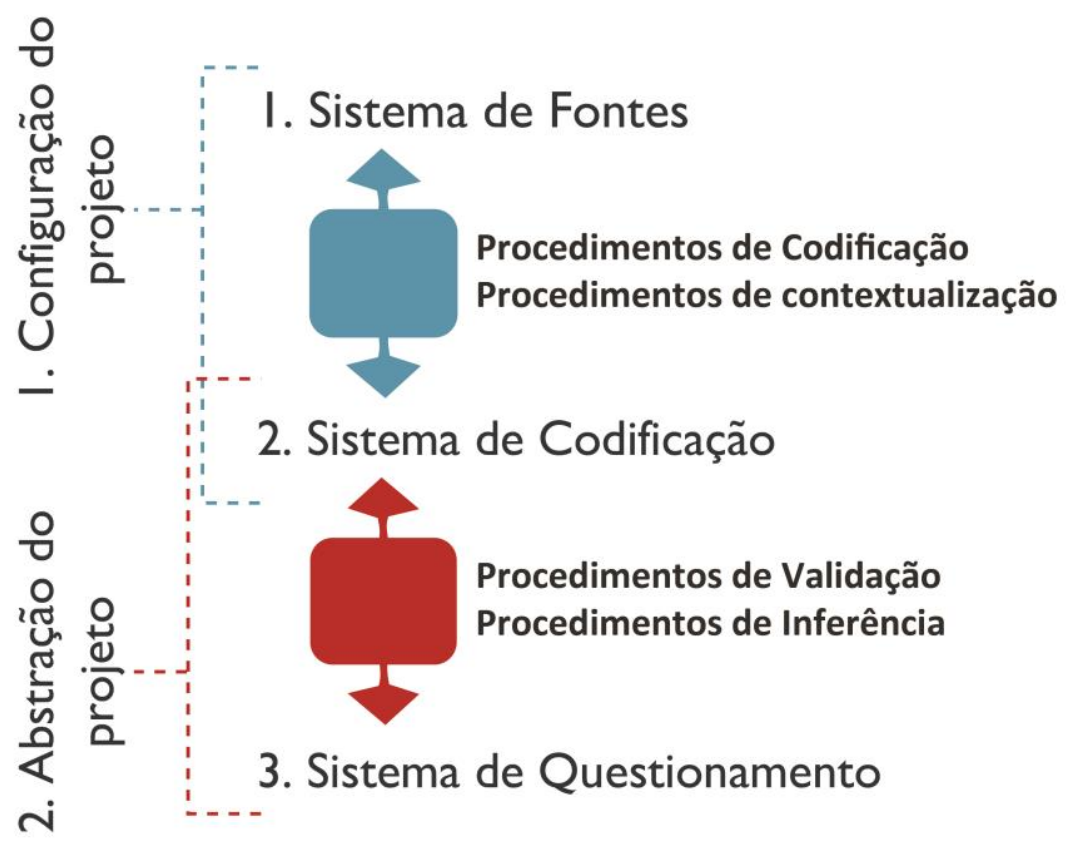

$\mathrm{Na}$ primeira parte o utilizador do webQDA necessita configurar o projeto inserindo informações no sistema de fontes e no sistema de codificação. Portanto, o processo de análise com a integração do webQDA é muito semelhante aos processos sem o uso de um sistema como este. É necessário transcrever, fazer leituras flutuantes, leituras recorrentes, codificação e recodificação, validação com outros investigadores etc.

Numa segunda fase, é possível questionar o sistema sobre os dados codificados, de tal maneira que o sistema do webQDA está preparado para "dar" respostas a perguntas específicas do utilizador na procura de padrões, matrizes e sínteses dos dados codificados. Nesta fase é possível abstrair da triangulação de todos os elementos do sistema processos de inferências e validação, aumentando a credibilidade e a consistência interna da análise.

Embora estejamos apresentando de forma linear, este processo pode ser realizado usando ferramentas que estão disponíveis na parte de questionamento antes mesmo de se utilizar o sistema de codificação. Exemplo disso são as ferramentas de busca de palavras ou 
expressões chave. Esta busca pode facilitar o processo de codificação em muito dos métodos de codificação disponíveis.

Foi objeto das formações compreender todos os detalhes da parte estrutural e processual da utilização do webQDA. Neste artigo, estamos interessados em compreender as dificuldades na formação e nestas partes fundamentais do webQDA.

\section{ANÁLISE DOS RESULTADOS}

Uma das linhas de argumentação que escolhemos neste artigo é a comparação constante entre os dados numéricos analisados e já publicados (NERI DE SOUZA; COSTA E NERI DE SOUZA, 2012) e o aprofundamento da análise qualitativa com base nos dados não-numéricos. Por isso, na apresentação dos resultados acerca das questões abertas do questionário, é oportuno salientar a posição dos formandos sobre a questão fechada: "Depois do curso de formação posso descrever as limitações e potencialidades do webQDA". Os resultados sinalizam que $73 \%(n=43)$ concordaram que tinham condição de descrever as limitações e potencialidades do software, comparativamente a 20,3\% (n=12) que se posicionaram indiferentes quanto a este item. Este é certamente um indicador de segurança sobre a aprendizagem realizada, mas quais são exatamente as potencialidades e limitações apresentadas? Para isso, as questões abertas foram: i) Qual a principal potencialidade que realça no webQDA? Porquê? e ii) Que limitações identifica no webQDA? Porquê?

Tabela 1 - Perceção dos Formandos por Sexo

\begin{tabular}{ccc}
\hline Sexo & Praticidade & $\begin{array}{c}\text { Sistematização e } \\
\text { Resultados }\end{array}$ \\
\hline Masculino & 5 & 9 \\
Feminino & 26 & 34 \\
Total & 31 & 43 \\
\hline
\end{tabular}

Emergiram da percepção dos formandos três importantes dimensões, i) as Potencialidades, ii) Limitações e iii) Sugestões. No entanto, consoante a proposta deste artigo, concentraremos a apresentação dos resultados nas potencialidades e limitações.

Ao levar a efeito a dimensão "Potencialidades", despontaram da leitura dos dados algumas categorias. No entanto, de acordo com os resultados da Tabela 1, as que se tornaram mais patentes para os formandos foram a i) "Praticidade" e a ii) "Sistematização dos Resultados". Relativamente a Categoria Praticidade, foram codificadas 31 unidades de texto, 
neste caso, 31 indivíduos reconhecem a praticidade do webQDA em termos de promover uma maior rapidez e optimização no tempo de análise dos dados. Podemos aferir esta categoria com algumas falas.

"A rentabilização do tempo empregue na análise dos dados."
"Permite um rápido e seguro tratamento de um grande volume de
entrevistas...."
"Porque muitas das fases da análise qualitativa da informação recolhida pode
ser efetuada no webQDA mais rapidamente e sem necessidade de um sem
número de folhas e papéis que podem ser destruídos em qualquer momento."

No que concerne a categoria "Sistematização dos Resultados", verificamos (Tabela 1) que na opinião dos formandos (43 referências) a sistematização dos dados, definição das categorias, codificações e obtenção dos resultados tornam-se uma mais-valia para a análise segundo estes participantes. Podemos exemplificar através de algumas respostas.

"O fato de otimizar o tempo, qualidade e precisão na sistematização dos dados."

"Permitir uma análise sistematizada e a manipulação de dados que de outro modo seria difícil e passível de falhas do que se faz ou do que fica por fazer."

"Categorização e análise de textos, vídeos, e áudio. Devido a enorme complexidade que esse trabalho exige. Desta forma tudo fica mais organizado e se cruza com mais facilidade."

Para além da dimensão "Potencialidades", julgamos necessário por em relevo a dimensão "Limitações". Destacamos como principal categoria de análise o "Sistema de Funcionalidade dos Comandos". Segundo as 26 referências registadas acerca da perceção dos formandos, esta categoria refere-se como limitações: i) a dependência do acesso à internet para aceder ao software, ii) exportação de alguns tipos de documentos que não são compatíveis com o sistema e iii) ainda não está formatado para pessoas com necessidades especiais. Elegemos algumas falas para exemplificar.

"Já referi anteriormente que o acesso exclusivo pela internet tem grandes vantagens, mas também limitações no que respeita à necessidade de estar ligado e também a questão de segurança".

"Juventude do software neste momento é a sua principal limitação e a acessibilidade para pessoas com necessidades especiais."

"Impossibilidade de exportação. Não caber todo o "mundo" da investigação, o que implica trabalho em dois sítios - computador e programa, sem que ose possa usufruir destes dados sem ser através do programa ou das impressões nos casos em que são possíveis".

Embora muitas das limitações apontadas ao webQDA tenham sido ultrapassadas poucos meses depois desta formação, é importante perceber as entre linhas dos discursos de alguns dos formandos. Como nesta última citação, que se pode perceber uma certa frustração

Comun. \& Inf., Goiânia, GO, v. 17, n. 2, p. 104-118, jul./dez. 2014. 
por se ter que "trabalhar em dois sítios - computador e programa". Uma das dificuldades no âmbito da formação foi justamente mostrar que todos os QDAS são ferramentas de apoio a análise dos dados, não existindo inteligência artificial para a tomada de decisão analítica. A ansiedade para que tudo seja "feito" no software também foi percebido por outros investigadores iniciantes nestas formações.

Outra dimensão que constitui uma das declaradas vantagens do webQDA é o facto dele funcionar em ambiente distribuído. Aproximadamente $84 \%$ concordam com a frase "compreendo as vantagens do webQDA funcionar online". No entanto, quando se analisa as questões abertas podemos perceber que os investigadores gostariam de ter uma versão offline do webQDA com a possibilidade de sincronizar depois com a nuvem de computadores. Esta aparente contradição entre ser uma vantagem ou desvantagem o facto dosoftware funcionar online, pode ser facilmente esclarecido com a análise das questões abertas e complementam a informação obtidas na escala de likert("Reader-focused text evaluation. An overview of goals and methods," 1997).

Quando avaliamos a formação webQDA propriamente dita 100\% dos formandos concordaram que "A formação webQDA contribui para uma visão de rigor e sistematização da análise qualitativa" e que "a formação webQDA facilita a compreensão da dimensão prática da investigação qualitativa"("Reader-focused text evaluation. An overview of goals and methods," 1997). Quando aprofundamos a análise complementando com as questões abertas, foi possível perceber três focos de sugestões para a melhoria da formação: i) aumentar o tempo de formação, ii) foca de forma mais profunda a fase do "Questionamento" que existe no webQDA, iii) propiciar acompanhamento e apoio após a formação. A seguir citamos algumas destas sugestões de melhoria:

\footnotetext{
"Instalar uma Assessoria Permanente pós formação para auxiliar os pesquisadores no uso do software para a realização da análise dos dados de suas pesquisas".

"Na fase do questionamento dada a formação incipiente ainda não domino esta etapa".

"Penso que o tempo de formação foi muito curto. É um software interessante mas complexo, necessita de mais prática para ser bem compreendido e, portanto, optimizado numa investigação".

"A formação está muito bem desenhada e conduzida, e não há dúvidas quanto à excelente qualidade. Somente partilharei convosco uma sensação que tive ao fim da formação. Por já estarmos um bocado cansados, acrescido à restrição temporal que obriga os formadores a avançar para a conclusão da sessão, o questionamento acabou por ser não tão bem aproveitado. Acho que algumas luzes do questionamento ficariam bem "mescladas" na parte inicial da formação. Não sei se é possível trabalhar o questionamento antes do fim. Caso não seja, sugiro uma gestão mais apurada do tempo, para que os
} 
formandos e formadores possam explorar com mais profundidade esta parte tão essencial, onde a análise toma corpo e ganha sentido".

A estrutura do webQDA é composta por três partes fundamentais: i) Fontes, ii) Codificação e iii) Questionamento(Neri de Souza, Costa \& Moreira, 2010; Neri de Souza et al., 2011; Costa, Linhares \& Neri de Souza, 2014). Em todas as formações, mesmos as mais avançadas e com maior tempo é possível constatar a dificuldade expressa pelos participantes destas formações iniciais - a fase do Questionamento. É nesta fase que é solicitado ao investigador o poder de síntese, triangulação e construção detalhada da coerência interna da análise. A fase do Questionamento através das pesquisas de código e do cruzamento das matrizes é uma fonte de dificuldade que vai para além das dificuldades com o próprio software, mas se remetem à natureza da construção do conhecimento nesta área.

Tudo o que apresentamos pode ser cruzado com o que foi obtido com as questões fechadas. Relativamente à posição dos inquiridos acerca da utilização do software webQDA, podemos inferir que $88,1 \%(n=52)$ concordam que após a formação tinham conhecimento suficiente para introduzir os dados e criar as categorias no webQDA, 73\% (n=43) tinham capacidade de descrever as limitações e potencialidades do software, comparativamente a 20,3\% ( $n=12)$ que foram indiferentes quanto a este item. É de referir o consenso dos respondentes $(100 \% ; n=59)$ no que toca a formação facilitar a compreensão da dimensão prática da investigação qualitativa. Nesta mesma direção, os resultados apontam em 98,3\% $(n=58)$, contrário a 1,6\% $(n=1)$ a concordância dos inquiridos sobre a formação do webQDA contribuir para uma visão de rigor e sistematização da análise qualitativa.

Tabela 2 - Posição dos inquiridos acerca da utilização do software webQDA

\begin{tabular}{|c|c|c|c|}
\hline \multirow[t]{2}{*}{ Itens } & \multicolumn{3}{|c|}{ Resumo da Escala de Likert } \\
\hline & Discordo & Indiferente & Concordo \\
\hline $\begin{array}{l}\text { Após o curso de formação tenho conhecimento suficiente } \\
\text { para introduzir os dados e criar as categorias no webQDA. }\end{array}$ & $0,0 \%$ & $0,0 \%$ & $88,1 \%$ \\
\hline $\begin{array}{l}\text { Depois do curso de formação posso descrever as limitações e } \\
\text { potencialidades do webQDA. }\end{array}$ & $0,0 \%$ & $20,3 \%$ & $73 \%$ \\
\hline $\begin{array}{l}\text { Compreendo que a formação webQDA facilita a } \\
\text { compreensão da dimensão prática da investigação } \\
\text { qualitativa. }\end{array}$ & $0,0 \%$ & $0,0 \%$ & $100 \%$ \\
\hline $\begin{array}{l}\text { A formação webQDA contribui para uma visão de rigor e } \\
\text { sistematização da análise qualitativa. }\end{array}$ & $1,7 \%$ & $0,0 \%$ & $98,3 \%$ \\
\hline
\end{tabular}

A perceção referida pelos inquiridos acerca da utilização do software na análise qualitativa dos dados, os resultados salientam às possibilidades da análise qualitativa serem maiores com o webQDA do que sem o uso do software, 95\% $(\mathrm{n}=56)$ dos respondentes concordaram. Seguindo na mesma direção, $88 \%(n=52)$ dos inquiridos, concordaram ao 
afirmar que a estrutura do software é de fácil compreensão. Sobre o uso do software, 81,3\% $(\mathrm{n}=48)$ concordaram que o mesmo é indispensável para a análise qualitativa dos dados, enquanto $13,5 \%(n=8)$ ficaram indiferentes e 1,6\% $(n=1)$ discordaram parcialmente. No entanto, um outro aspecto que é digno de nota foi saber dos inquiridos se pretendiam usar o webQDA nas suas investigações, 89,8\% (n=53) confirmaram esta opção, 5\% (n=3) ainda não decidiram e $1,6 \%(n=1)$ negaram.

Tabela 3 - Posição dos inquiridos acerca da utilização do software na análise qualitativa

\begin{tabular}{|c|c|c|c|}
\hline Itens & \multicolumn{3}{|c|}{ Resumo da Escala de Likert } \\
\hline & Discordo & Indiferente & Concordo \\
\hline $\begin{array}{l}\text { Considero a estrutura do software webQDA de fácil } \\
\text { compreensão. }\end{array}$ & 3,3 & $5 \%$ & 52) \\
\hline $\begin{array}{l}\text { Considero o uso do webQDA indispensável para a análise } \\
\text { de dados qualitativos. }\end{array}$ & $1,6 \%(1)$ & $13,5 \%(8)$ & $81,3(48)$ \\
\hline Pretendo usar o webQDA na minha investigação. & $1,6 \%(1)$ & $5 \%(3)$ & $89,8(53)$ \\
\hline $\begin{array}{l}\text { Considero que as possibilidades de análise qualitativa com } \\
\text { o webQDA são maiores do que sem o uso de software. }\end{array}$ & $1,6 \%(1)$ & $3,38 \%(2)$ & $95 \%(56)$ \\
\hline
\end{tabular}

\section{CONSIDERAÇÕES FINAIS}

A investigação qualitativa tem alcançado novos padrões de qualidade através da utilização de software específico para a análise de dados, os chamados Qualitative Data Analysis Software- QDAS(LINDA et al., 2014). No entanto, estes mesmos QDAS trazem desafios ao nível de usabilidade e de formação de investigadores que necessitam integrar as técnicas de análise com as potencialidades das QDAS.

Um dos desafios discutido neste artigo foi ao nível da formação de novos investigadores na conjugação de técnicas de análise e o software webQDA. Assim, este trabalho, apresentamos as perceções de investigadores sobre a utilização do software webQDA no contexto de uma formação avançada. Recorremos a uma análise qualitativa de questões abertas com a utilização do próprio webQDA, que foram recolhidas através de inquérito por questionário.

Os resultados apontaram para uma perceção positiva dos formandos acerca da utilização do webQDA e da própria formação. Analisamos as potencialidades e limitações do webQDA. Em termos de potencialidade foram apontadas a "Praticidade" e a "Sistematização dos Resultados" que o webQDA proporciona. As principais limitações apontadas foram: i) a dependência do acesso à internet para aceder ao software, ii) exportação de alguns tipos de documentos que não são compatíveis com o sistema e iii) ainda não está formatado para pessoas com necessidades especiais. Quanto a formação, sugerem que é necessário mais tempo para a dimensão do questionamento e cruzamentos dos dados com apoio do webQDA. 
Foi possível perceber também que a utilização do software é um elemento dinamizador de novas compreensões, competências e aprofundamentos sobre o processo de análise qualitativa de dados.

Qual será o futuro do sistema de análise qualitativa de dados? Consideramos que as ferramentas disponíveisatualmente na estrutura no webQDA são suficientes em todo o processo de investigação qualitativa. Contudo,acreditamos que muitos algoritmos e processos de inteligência artificial poderiam ser implantadosnestes sistemas com ganho para a qualidade da investigação nas ciências humanas e sociais. Outras funcionalidades que façam mais facilmente a interfase com os processos qualitativos e quantitativos em geral poderá ser desejáveis principalmente em investigações de natureza mista. É difícil prever plenamente onde as tecnologias poderão nos levar neste âmbito, mas temos a certeza que a informatização destes processos são fundamentais para uma sociedade que deseja obter resultados de qualidade e credibilidade elevada nas ciências.

\section{REFERÊNCIAS}

CARRERA-FERNÁNDEZ, María Jesús; GUÀRDIA-OLMOS, Joan; PERÓ-CEBOLLERO, Maribel. Qualitative methods of data analysis in psychology: an analysis of the literature. Qualitative Research, Barcelona, v. 14, n. 1, p. 20-36, nov. 2012.

INTERNATIONAL ORGANIZATION FOR STANDARDIZATION. ISO/IEC9126. Avaliação de Qualidade de Produtos de Software . Geneva: International Standards Organisation. [s.i.], 1999.

LINDA, S. G.; KRISTI, J.; GREGORIO, S. di. Tools for analyzing qualitative data: the history and relevance of qualitative data analysis software. In.: SPECTOR, J. M. et al (Eds.), Handbook of Research on Educational Communications and Technology. 4. ed. London: Springer, 2014. p. 221-236.

NERI DE SOUZA, D.; COSTA, A. P.; NERI DE SOUZA, F. Avaliação da Perceção dos Formandos sobre o Software WebQDA. In: Actas... II Congresso Internacional TIC E Educação (ticEDUCA2012). Lisboa: ticEDUCA, 2012a.

NERI DE SOUZA, D.; COSTA, A. P.; NERI DE SOUZA, F. Avaliação da Perceção dos Formandos sobre o Software WebQDA. In: Actas... II Congresso Internacional TIC e Educação (ticEDUCA2012). Lisboa, 2012b. p. 365-376.

NERI DE SOUZA, F.; COSTA, António Pedro; MOREIRA, A. Questionamento no Processo de Análise de Dados Qualitativos com apoio do software webQDA. EDUSER: Revista de Educação, Inovação Em Educação Com TIC, v.3, n. 1, p. 19-30, 2011.

NERI DE SOUZA, F.; COSTA, A. P.; MOREIRA, A. WebQDA: Software de Apoio à Análise Qualitativa. In.: ROCHA, A. (Ed.). Conferência Ibérica de Sistemas e Tecnologias de Informação, 5 CISTI'2010. Santiago de Compostela, Espanha: Universidade de Santiago de Compostela, 2010.

Comun. \& Inf., Goiânia, GO, v. 17, n. 2, p. 104-118, jul./dez. 2014. 
NERI DE SOUZA, F.; COSTA, A. P.; MOREIRA, A. WebQDA: manual do Utilizador. 2. ed.. Portugal: Universidade de Aveiro, 2013.

DEJONG, Menno; SCHELLENS, Peter Jan. Reader-Focused Text Evaluation: An Overview of Goals and Methods. Journal Of Business And Technical Communication, [s.i.], v. 11, n. 4, p.402-432, out. 1997.

Recebido em: 13/09/2014

Aceito em: 30/10/2014

Publicado em: 10/12/2014 